Published as: Maryam Asrar, Amin Al-Habaibeh, Bubaker Shakmak, Sally Jane Shaw, "A device for improving the visual clarity and dimension of veins", British Journal of Nursing | Vol. 27 | No. 19 | pp S26-S36; https://doi.org/10.12968/bjon.2018.27.19.S26

\title{
A device for improving the visual clarity and dimension of veins
}

\author{
M. Asrar ${ }^{1}$, A. Al-Habaibeh ${ }^{2, *}$, B. Shakmak ${ }^{3}$, S. J. Shaw ${ }^{4}$ \\ ${ }^{1,2,3}$ Department of Product Design, Nottingham Trent University, Nottingham, UK. \\ www.ntu.ac.uk/adbe \\ ${ }^{4}$ VIP Venepuncture \& Cannulation Training, UK. www.vipvenepunctureandcannulation.co.uk \\ *Corresponding author. E-mail: Amin.Al-Habaibeh@ntu.ac.uk
}

\begin{abstract}
Vascular access for venepuncture and peripheral intravenous cannulation is a common procedure within healthcare. This research investigates the effectiveness of an innovative device called the Vacuderm in increasing vein dimensions, temperature difference between vein and its surrounding and visual clarity through an additional effect of creating a vacuum on top of the tourniquet. A randomized cross over design is used in this study for looking at the vein visibility, dimensions and thermal behaviour using infrared thermography. Both dorsal hands are assessed in a random cross over order of 20 healthy volunteers with and without the application of the Vacuderm. The results show significant increase in venous diameter and venous cross sectional area with highly significant increase in vein clarity caused by using the Vacuderm which creates a negative pressure for transient suction in addition to its vein occlusion effect.
\end{abstract}

Keywords: Infrared thermography; Intravenous cannulation; Veins; Tourniquet; Venepuncture. 


\section{Introduction}

Many patients attending hospitals require either venepuncture for blood samples or peripheral intravenous cannulae (PIVC) to facilitate the administration of intravenous medication. Essential to successful vascular access is vein location, assessment and optimum preparation for cannulation. ${ }^{1}$ It has been stated that over a billion PIVCs are inserted each year in hospitalised patients worldwide. ${ }^{2}$ While the volume of PIVCs being inserted worldwide may warrant further study, a major concern for practitioners and patients is difficult venous access (DiVA). Difficult venous access can be defined as non-visible and non-palpable veins requiring highly experienced practitioners to use vein locating technology aids to insert a vascular access device (VAD). ${ }^{3}$

\section{Difficult Venous Access}

The factors that contribute to DiVA relate to both the patient and practitioner. If the practitioner lacks experience and/or knowledge this can lead to poor assessment of the veins ${ }^{4}$ or placement of a VAD in a suboptimal site such as the anti-cubital fossa or the back of the hand. Placement of cannulae at areas of flexion increases the risks of complications such as phlebitis and cannula failure ${ }^{5}$, these sites are commonly used in DiVA patients. ${ }^{6}$ Lack of awareness of vein location aids and how to use them or access to these aids also contribute to DiVA.

Patient factors that contribute to DiVA making the vein difficult to palpate or visualise include deeper vein location due to obesity, skin pigmentation, tattoos, oedema and patient medical history such as chemotherapy treatment. ${ }^{7}$ The age of the patient can impact on venepuncture and cannulation; due to the ageing process the vein structure becomes weaker, due to loss of collagen and weaker endothelium ${ }^{8}$ meaning adequate assessment of the veins is essential. It has been found that older patients have reduced vasculature suitability for PIVC leading to multiple insertion attempts. ${ }^{9}$

When patients have DiVA this increases the risk of first attempt cannulation failure. Each failed attempt has an impact on the patient and on the risks to further cannulae placement. It has been found within an emergency department patients reported an increase of pain with each failed cannulation attempt when multiple attempts had been made; stating that cannulation is the most painful aspect of their treatment. ${ }^{10}$ It has been described that the effect of repeated failed attempts of cannulation can lead to loss of an access site or it being compromised and the risk of subsequent phlebitis and failure is increased. ${ }^{11}$ Researchers have 
found that staff reported attempting DiVA multiple times ending up placing the PIVC in a small vein which had inadequate blood flow which causes the cannula to fail just a few hours later. ${ }^{12}$ The Royal College of Nursing Standards ${ }^{13}$ for infusion therapy places the focus of vein assessment and selection on preservation of the vessel by recommending that organisations should consider the use of a Vessel Health and Preservation Framework (VHP) to support practitioners in the assessment and selection of the most appropriate vessel, VAD, at the right time. ${ }^{4}$ This places the focus of vascular access on the assessment and correct VAD placement rather than the mind-set of just get a cannula in any vein.

Astonishingly, limited literature has been found focusing on the patient characteristics and visible vein count in the difficult venous access cases. Many patients' characteristics have been reported in the previous studies associated with difficult venous access for venepuncture and cannulation, but the investigative studies are very limited about these characteristics. ${ }^{14-18}$ However, Researchers have investigated patient characteristics and factors affecting vein visualization and found that African-American or Asian ethnicity, and obese weight are associated with poor vein visibility. ${ }^{19}$

As has been discussed above, difficult venous access is a reality, leading to delays in patient treatment. The RCN IV Standards ${ }^{13}$ focuse the practice on vessel health and preservation (VHP). The recent developments in vein location aids such as near infrared vein viewers, ultrasound and novel tourniquets and vascular access devices supports VHP. Moreover, researchers have developed several safer techniques for vein cannulation such as using ultrasound guidance and imaging. ${ }^{20,21}$

This paper will describe and discuss a research investigation of a novel tourniquet, the Vacuderm $^{\mathrm{TM}}$, which is designed to improve venous distension and filling through a manual pumping mechanism that creates vacuum or negative pressure which amplifies the effect of venous occlusion by its tourniquet effect. This is the first study for this particular device.

Inserting a needle into a vein is amongst the most commonly performed invasive medical procedures globally. Needle insertion could be for several different reasons, either IV cannulation (i.e. anaesthesia, drugs delivery, medication, fluids or blood components administration) or blood sampling (phlebotomy). First attempt cannula insertion success rate is found to be lower in some patient groups such as the elderly, children, obese and darker skinned patients. The patients could be at higher risk of getting infection as a result of multiple unsuccessful cannulation attempts. This ultimately causes increased stress and a traumatic experience for both patients and practitioners. 
To improve the first attempt success rate for venous access, the Vacuderm ${ }^{\mathrm{TM}}$ has been designed as a single-use disposable tourniquet. The Vacuderm has been designed to aid intravenous needle insertion for cannulation, blood collection and drug delivery through pumping action in addition to the tourniquet effect.

\section{The Vacuderm: structure and use}

The Vacuderm is a device designed for improving vein preparation to increase ease of venepuncture and cannulation in situations of difficult venous access. It is a tourniquet with an integrated dome designed to create a transient vacuum or negative pressure via manual pumping to inflate the vein further and amplify the effect of vein occlusion in order to improve ease of cannulation. The vacuum pump amplifies its tourniquet effect through a suction and pump mechanism which makes the vein more tense for easier venous access via the negative pressure. The additional pumping of the dome is intended to improve the tension and size of the vein, usually assessed by palpation and also the visualisation of the vein. The Vacuderm is made to have a soft strap and thicker material dome for increased force of pumping. The vacuderm (total length: $415 \mathrm{~mm}$ ) is composed of a strap (300mm x 15mm), dome (29.4mm x 42mm x 15mm) and buckle (50mm x 22mm), as shown in Fig. 1.

This study investigates the physiological effects of a novel tourniquet, the Vacuderm, on the vein dimensions, temperature, and visual clarity through pumping action in addition to the tourniquet effect using infrared thermography. We hypothesised that pumping effect in addition to the tourniquet effect of the Vacuderm would be associated with finding more visible, prominent, expanded in diameter and higher in temperature veins. 

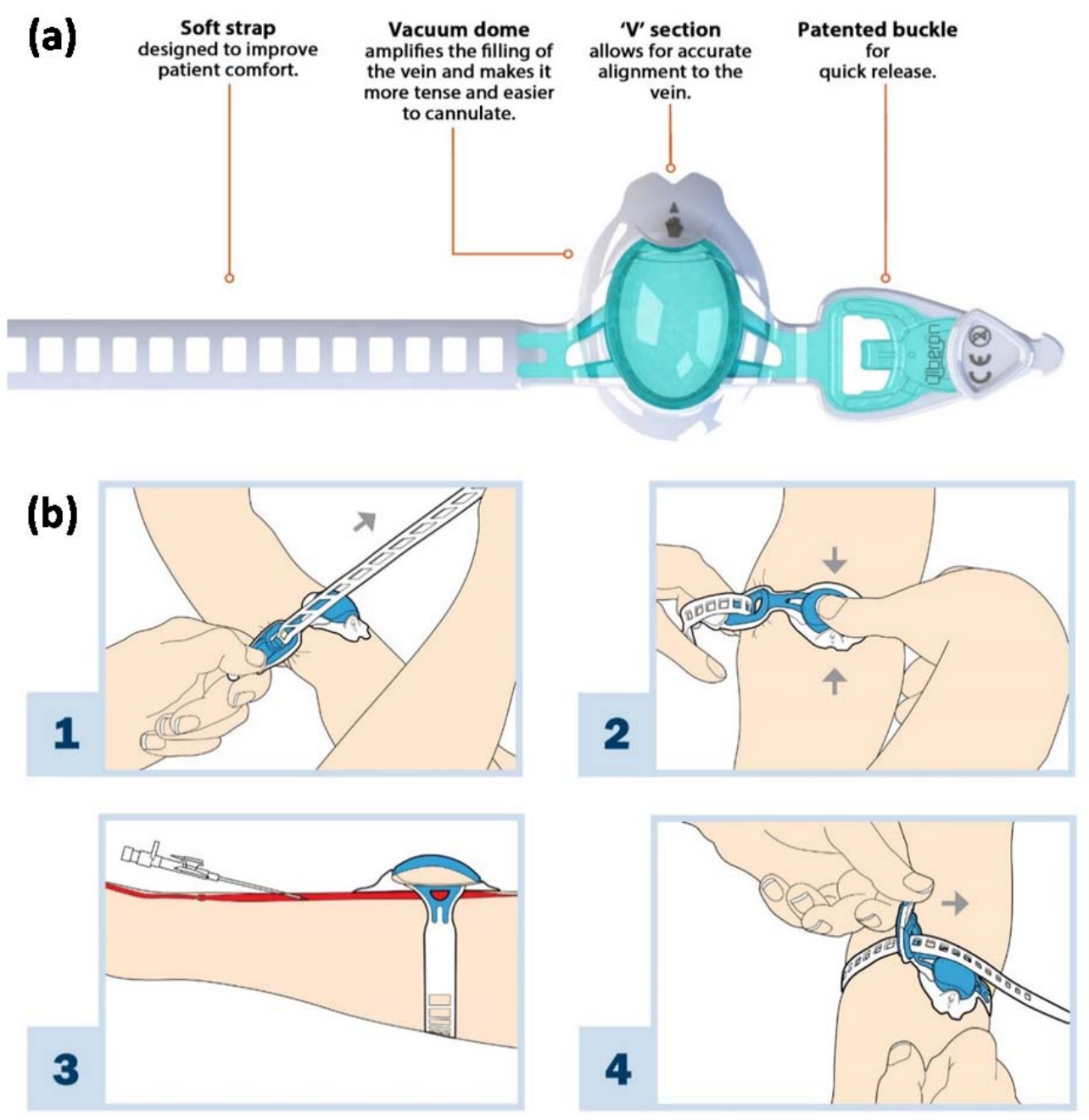

Fig 1 (a) The Vacuderm ${ }^{\mathrm{TM}}$, (b) the instructions to use Vacuderm; (1) thread the strap through the buckle and stretch, ensuring ample tightening to latch onto the buckle, (2) pump at least 10 - 15 times to optimise vein distension, (3) insert the cannula as per standard practice and (4) release by lifting the buckle. $^{22}$ 


\section{Research Methodology}

To test the efficacy of Vacuderm in relation to vein visibility and clarity, 20 subjects responsible for generating 40 sets of data for the dorsum of right and left hands and a total of 360 thermal images are included in this study. For reliability of the research outcomes, both upper extremities of 20 volunteers i.e. 12 male and 8 female are involved in this research.

The experimental set-up, consists of infrared vein locating system, cold compress, the Vacuderm tourniquet, stop watch, weighing scales, metallic tape measure, thermometer, and laptop for data collection, acquisition and analysis (Fig. 2-a).

A total of 20 healthy volunteers are enrolled onto the trial after giving their written consents. Ethics committee approval is obtained from the Nottingham Trent University Research Ethics Committee. Participants' age, skin tone, height, weight, body mass index, gender, and ambient temperatures before and after the experimental procedure are measured and recorded for the purpose of this study (Supplementary Table A1). 

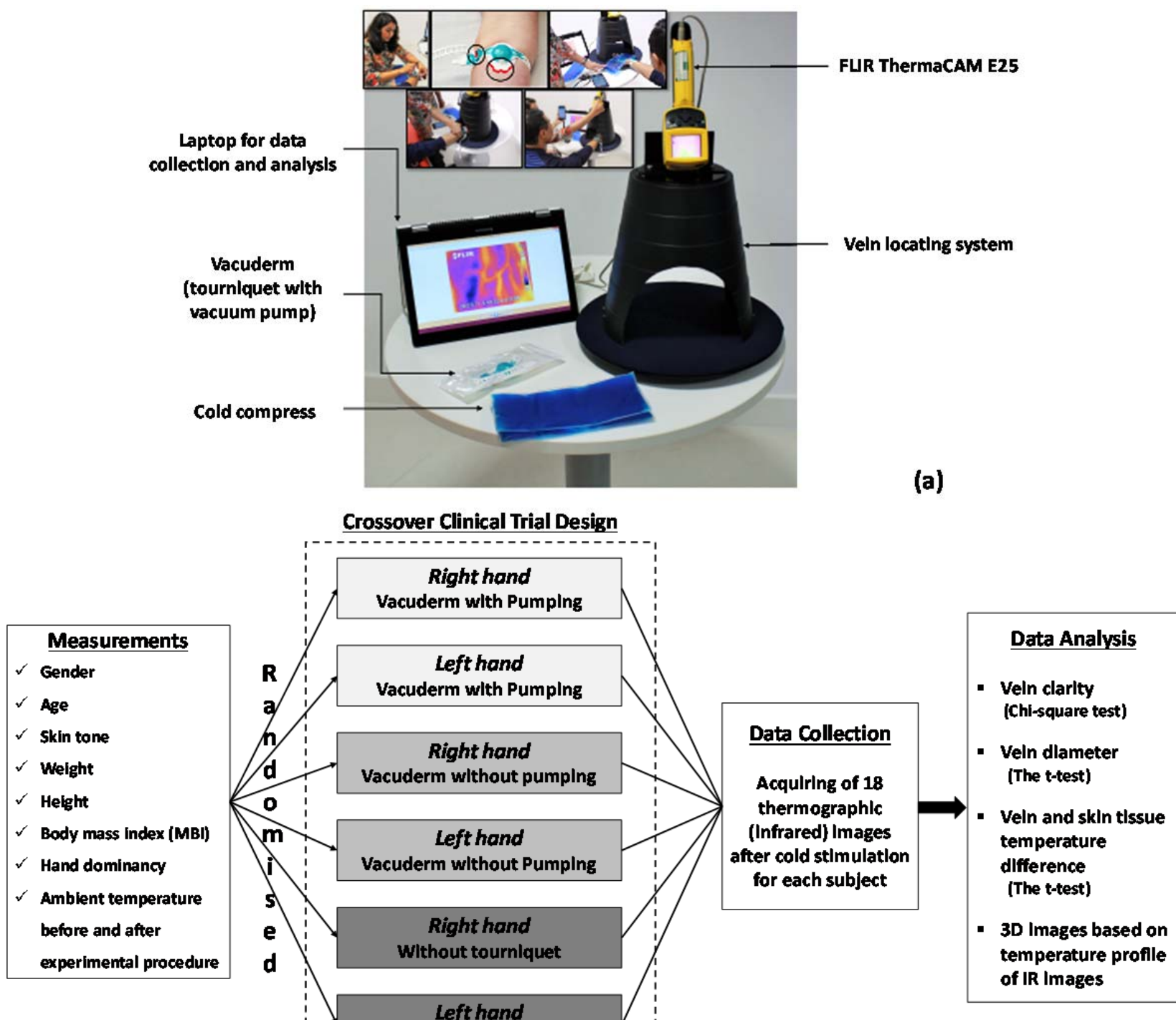

Without tournlquet

(b)

Fig 2 (a) The experimental set-up and (b) a randomised crossover design for the measurement of patient characteristic factors for vein visualization and testing the efficacy of the Vacuderm.

A randomized cross over design is used in this study for looking at the vein visibility using infrared imaging, as shown in Fig. 2-b. Both dorsal hands are assessed in a random order with and without the application of the Vacuderm. The Vacuderm tourniquet is applied just proximal to the wrist in the distal forearm. In the case of the application of the Vacuderm, two situations are assessed, namely pumping and non-pumping in a randomized order again. When pumping, 15 times pumping of the dome is made for all subjects. All other variables are kept constant. To keep all other variables constant, the position of the initial application of the dome on forearm/wrist is marked so reapplication for the cross over measurement must be made on the same place. To ensure the same level of tightening of the Vacuderm for pumping and non-pumping, the position of the notch on the Vacuderm used for latching the 
catch is also marked so the same level of tightening should be exercised at pumping and nonpumping for each arm (Fig. 2-a). All subjects are asked to be hydrated before the procedure. All sets of data are captured in the seated position of the subjects while resting their arms straight on the base of the vein locating system. Six cold compresses are used for each step, which are maintained at the same temperature in a refrigerator, for each individual and are taken out of fridge just before the start of each step. Prior to each measurement the arm is raised for 5 seconds to empty the vein and a cold compress is applied for 15 seconds. The time from application of the cold compress to the start of the acquisition of 3 infrared images is kept constant for all three scenarios, such as without tourniquet, pumping and nonpumping. The data in the form of thermal images are collected for the three scenarios. Same scenarios are repeated alternatively on both dorsal hands in a randomised order. Therefore, six sets of data in the form of a total of 18 thermal images are captured for both dorsal hands of each participant, three sets for each hand and each set consisting of three thermal images with an interval of $10 \mathrm{~s}$.

Temperature difference between veins and skin tissue, vein diameter and vein clarity are estimated with the application of the Vacuderm as a tourniquet without pumping and comparing it with the application of the Vacuderm with pumping.

It is worth noting that the back of the hand is used as the study is focused on showing the veins change at a longer distance from the Vacuderm to ensure the effect is significant even at the distal aspect of the limb and the ease of accessibility for infrared imaging. However routine cannula placement is discouraged in the back of the hand and aspects of the limb subjected to joint flexion such as the elbows or the wrists. 


\section{Results}

Three steps as presented in Supplementary Table A2 are performed to collect infrared images of all subjects. Six sets of data in the form of thermal images are captured for both dorsal hands of each participant, three sets for each hand (each set consisted of three thermal images with an interval of $10 \mathrm{~s}$ ). All 18 thermal images for each subject are captured at the same time (i.e. at 25 seconds, $35 \mathrm{~s}$ and $45 \mathrm{~s}$ ) after $15 \mathrm{~s}$ long application of cold compress. Table A2 presents IR images (single thermal image of each condition) for a female and a male subject for right and left hand, respectively for all three situations involved in the current study.

\section{Statistical Analyses}

Three data analyses are performed on a total of 360 (18 x 20) infrared images. Temperature difference between veins and skin tissue, vein diameter and vein clarity are estimated with the application of the Vacuderm as a standard tourniquet (i.e. without pumping) and comparing it with the application of the Vacuderm with pumping. Statistical analyses with Chi-Square test and the paired T-Tests are also performed on the vein clarity grades, temperature difference values and vein diameter values.

\section{Clarity of veins}

Clarity refers to the degree to which the blurriness or indistinctness is present for the visualisation of the veins in the thermal image of a person's hand. It also refers to the degree to which the veins are identifiable or not with the naked eye.

All thermal images of right and left hands of each participant are graded for clarity using a 3 point vein clarity scale. In grading vein clarity, the principal investigator considers the number, size, colour, depth, sharpness, width, and position of every vein visible with the naked eye. Table 1 is the vein clarity chart with grade, sample infrared images and categories, accompanied by further explanatory comments. 
Table 1 Veins Clarity Chart

\begin{tabular}{|c|c|c|c|}
\hline Grade & Thermograms & Category & Explanatory comments \\
\hline 1 & & Excellent visibility & $\begin{array}{l}\text { Not only the superficial veins but } \\
\text { also the deeper veins are extremely } \\
\text { easy to identify with the naked eye. } \\
\text { The veins appeared either very } \\
\text { sharp, clear, distinct or expanded. }\end{array}$ \\
\hline 2 & & Good visibility & $\begin{array}{l}\text { At least the prominent veins are } \\
\text { easy to see with the naked eye with } \\
\text { no difficulty. }\end{array}$ \\
\hline 3 & & Poor visibility & $\begin{array}{l}\text { Eminently difficult to identify even } \\
\text { the prominent veins through the } \\
\text { naked eye. }\end{array}$ \\
\hline
\end{tabular}

Infrared images of right dorsal hand of a female subject are presented for the elaboration of grading in the above vein clarity chart for three different scenarios; without tourniquet (grade 1), with tourniquet plus pumping (grade 2) and with tourniquet but no pumping (grade 3). Mostly the thermal images captured without tourniquet are graded higher because of the sharp appearing veins with higher temperature differences between the veins and the surrounding tissues. The most obvious reason is the absence of disturbance in the venous outflow in the scenarios where no tourniquet is applied. However, the scenarios where the tourniquet is used, the compression is made sufficient to allow arterial inflow whilst limiting venous outflow. Due to limited venous outflow, the temperature differences between the veins and the surrounding tissues have been found to affect the clarity as compared to without tourniquet scenarios.

Therefore, the real comparison is between the scenarios where tourniquet is introduced but in one situation the pumping effect is absent, whereas in the other it is present. Supplementary Table A3 summarises the vein clarity grades for all subjects of the current study for left and right hands for all possible scenarios.

\section{Vein clarity analysis: $\chi 2$ calculations}

In the present study, the authors' main focus is to investigate the pumping effect of the Vacuderm, with the traditional tourniquets which involve only tightening but no pumping effects. Hence, in the statistical analysis we only compare pumping versus non-pumping; and dealing without tourniquet data just as a base for reference. 
Thus, two-way contingency yields highly significant results for the clarity of veins for right and left dorsal hands for pumping and non-pumping scenarios. The results prove that pumping has statistical significance over non-pumping for both hands in relation to the vein visibility (see Fig. 4-a1 and Fig. 4-a2).

The chi-square statistics for left dorsal hands is 10.1403 . The $p$-value is 0.006282 . The result is significant at $p<0.01$. Also, for the right dorsal hand $\chi^{2}=9.817, \mathrm{df}=2, \chi^{2} / \mathrm{df}=4.91, \mathrm{P}$ $(\chi 2>9.817)=0.0074$. The result for right dorsal hands is significant at $p<0.01$.

\section{Vein diameter analysis}

After selecting the region of interest in all thermal images, a prominent vein is chosen and for a precise measurement five times makers are placed at different positions on the same vein and by taking the average of those five diameters finally the most accurate diameter of that particular vein is calculated (see Fig. 3).

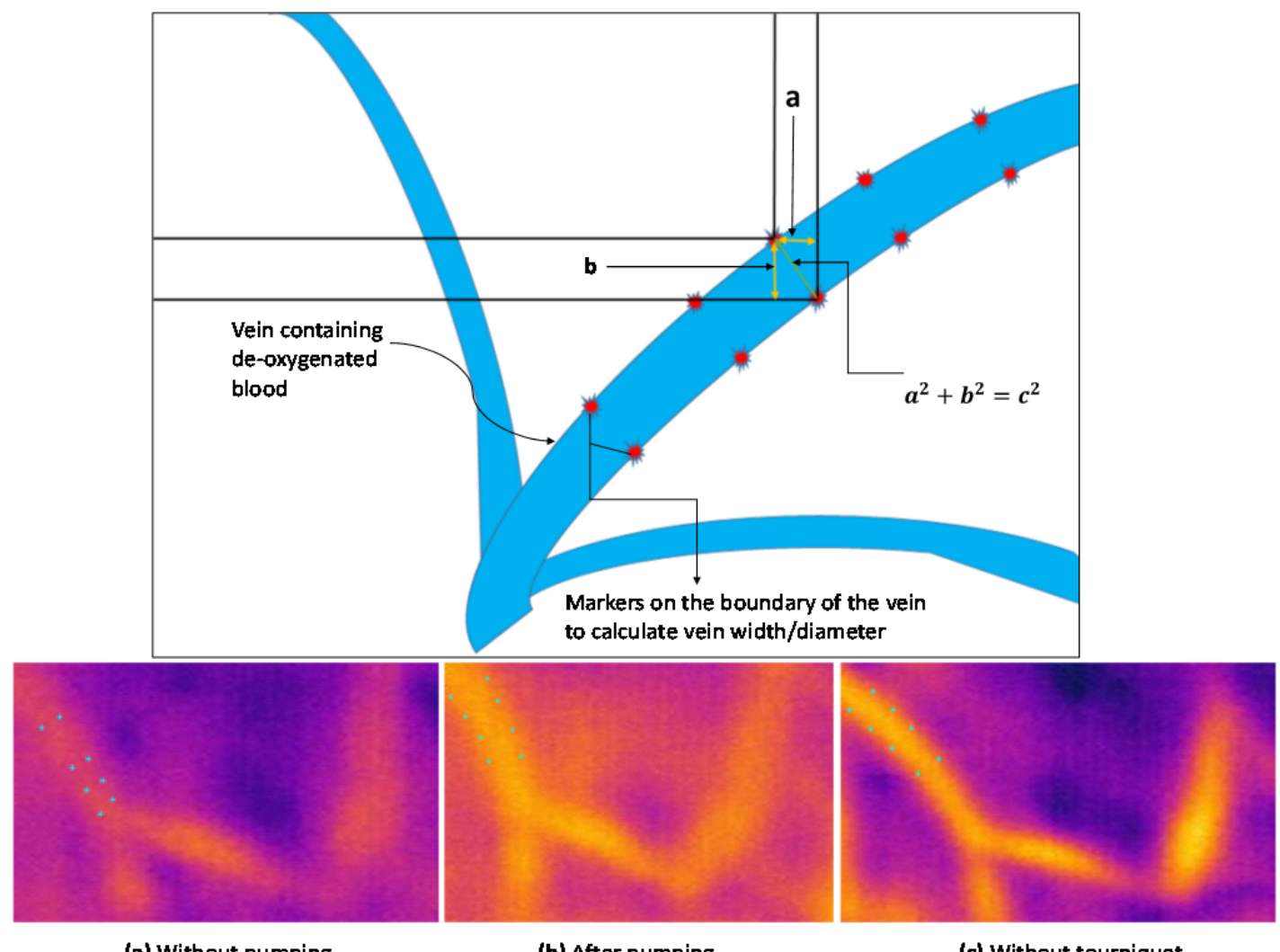

(a) Without pumping

(b) After pumping

(c) Without tourniquet

Fig 3 The vein diameter as illustrated in the schematic diagram is measured using the Pythagorean Theorem formula. Cyan colour markers are showing how diameter of the same vein in all three different scenarios for the same subject is measured. Thermal images of right dorsal hand of a female subject (a) after applying tourniquet and cold stimulation but no pumping, (b) after applying tourniquet, cold stimulation and pumping, (c) after applying only cold stimulation and no tourniquet. 
For right and left dorsal hands of each subject, the same procedure is repeated for three different scenarios i.e. without tourniquet, tourniquet on but without pumping and tourniquet on plus pumping effect seen in Fig. 3. Same segment of the vein is chosen for all three scenarios in order to know the change in the diameter of a particular portion of the vein.

It is clearly shown that pumping effect is playing a vital role, not only in the expansion of veins, but also in improving the visibility of the veins as compared to the situation where there is no pumping. As pumping permits the venous flow gradually, better contrasts are obtained between veins and the surrounding tissues based on thermal behaviour. Secondly, pumping clearly has helped in vein dilation. Pumping effect contributes by producing more tension in the veins due to which the diameter of veins expands eventually. The average calculated vein diameter and/or width values for the subject as presented in Fig. 3 are: 9.7 pixels (without pumping), 16.2 pixels (after pumping) and 12.9 pixels (without tourniquet). The highest obtained value is after pumping indicating that pumping is responsible of vein expansion and increase in the size of the diameter of the vein. The increase of 6.5 pixels $(40 \%)$ reveals a significant difference between vein diameter with and without pumping and indicates a remarkable enlargement in the size of the vein as a result of pumping.

\section{Vein diameter analysis: t-test analysis}

Fig. 4-b1 and Fig. 4-b2 summarise the results of all 20 volunteers for the vein diameter analysis for pumping versus non-pumping scenarios for both hands of each individual. Bar charts in Fig. 4-b3 and Fig. 4-b4 illustrate the overall result of the vein diameter analysis for pumping versus non-pumping for left and right dorsal hands, respectively. For the left hand, the mean score for pumping condition is much higher $(\mathrm{M}=14.84, \mathrm{SD}=1.74)$ than for the non-pumping condition $(\mathrm{M}=11.95, \mathrm{SD}=1.49)$. There is $24 \%$ increase in mean diameter and a $54 \%$ increase in the cross sectional area of the target vein with high statistical significance on paired t-test; $\mathrm{t}(19)=-8.234, \mathrm{p}<0.001$. For the right hand (as indicated in Fig. 4-b), the mean score for pumping condition is significantly higher $(\mathrm{M}=14.20, \mathrm{SD}=2.00)$ than for the non-pumping condition $(\mathrm{M}=10.86, \mathrm{SD}=1.78)$. There is a $31 \%$ increase in diameter with a $70 \%$ increase in the cross sectional area of the target vein with high statistical significance on paired t-test $\mathrm{t}(19)=-11.148, \mathrm{p}<0.001$. 


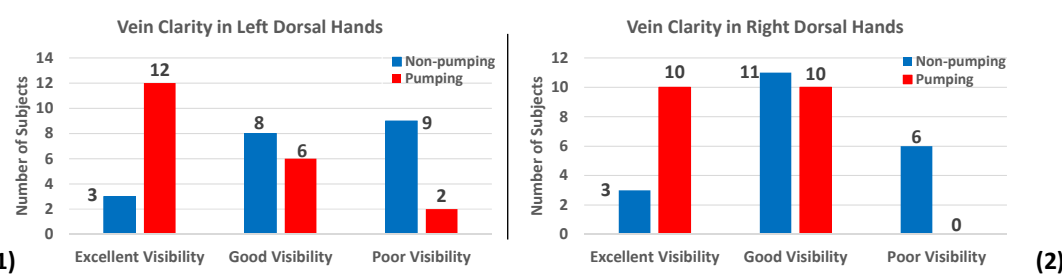

(a) Pumping has statistical significance over non-pumping for the vein visibility for both hands.

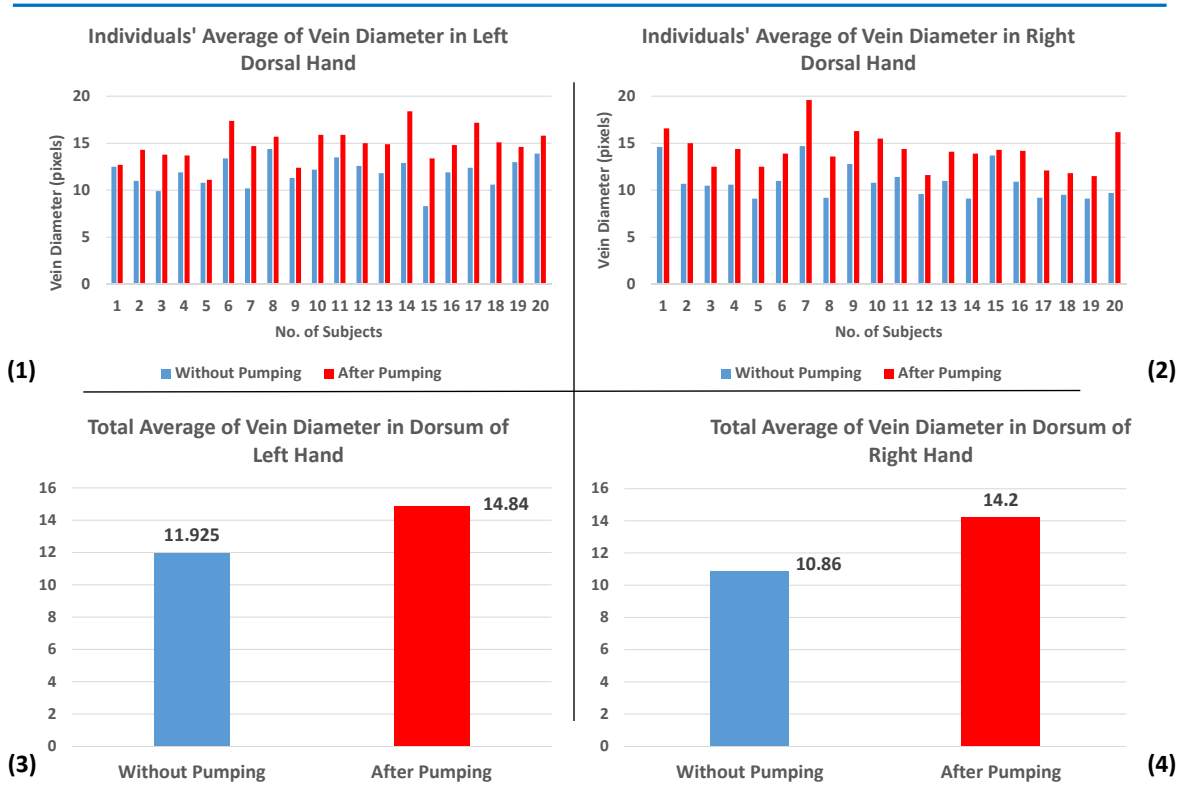

(b) Individuals' average of vein diameter in both left and right hands of all 20 subjects is increased considerably as a result of the pumping effect of the Vacuderm $(\mathbf{1} \& \mathbf{2})$. The total average of vein diameter for all subjects is increased significantly as a result of the pumping effect of the Vacuderm in both left and right hands (3 \& 4).

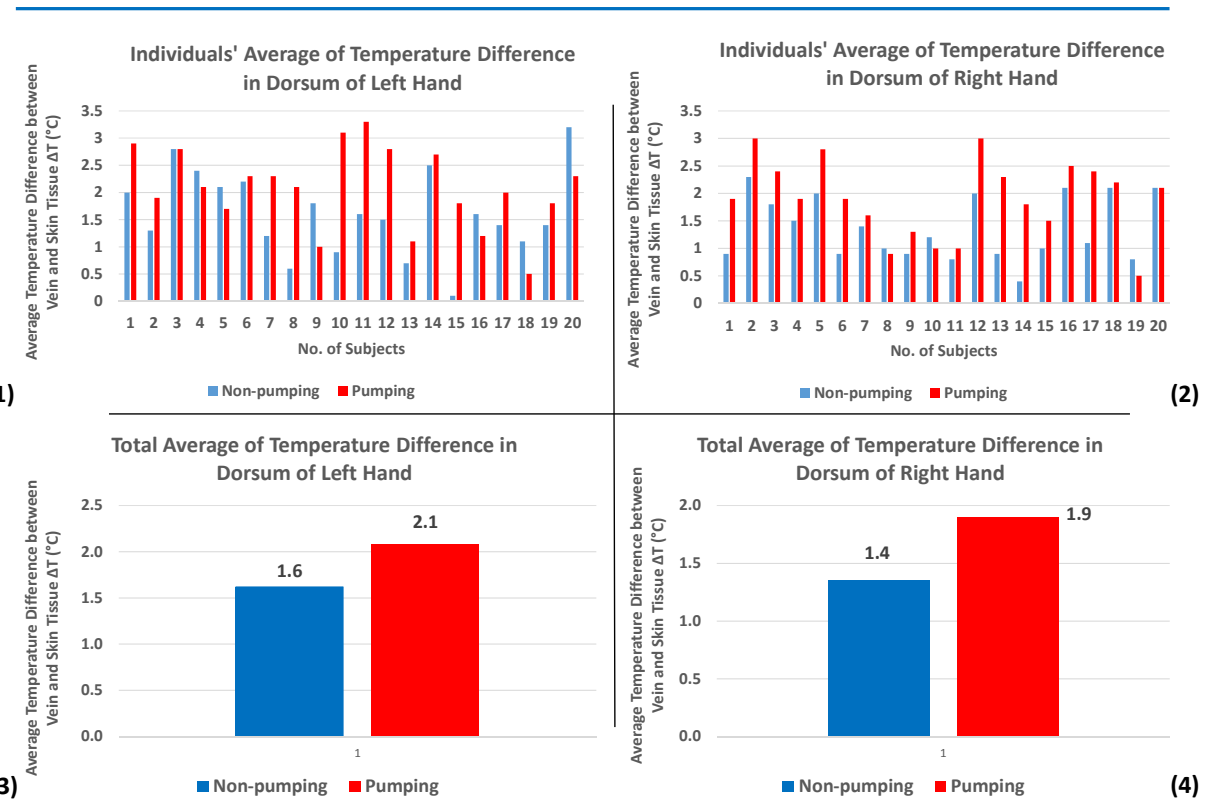

(c) The average temperature difference between the vein and the surrounding tissue after pumping and nonpumping conditions for all individuals in both left and right dorsal hands (1 \& 2). The total average of temperature difference between the vein and the surrounding tissue temperatures for all 20 subjects is increased by $0.5^{\circ} \mathrm{C}$ as a result of the pumping effect of the Vacuderm in the cases of both left and right dorsal hands (3 \& 4).

Fig 4 (a) Vein clarity, (b) vein diameter and (c) vein thermal behaviour in the cases of pumping and nonpumping of the Vacuderm for both hands of all subjects. 


\section{Vein temperature analysis}

Enhancement of thermal vein to tissue contrast is desired, in order to assess veins precisely using infrared thermography. The current study employs a novel approach to effectively enhance the skin vein to tissue contrast by using cold stimulations on skin surface. Infrared thermography has been proved to be a good technology to detect and enhance the visualization of veins especially when followed by cold stimulations. ${ }^{23}$ Also, it has been reported that the veins are clearly visible in the thermal image recorded after cold stimulation $^{24}$.

Thermal response of the skin and veins to the thermal stimulation is analysed. Temperature data is accessed by FLIR QuickReport. Five points are marked on the veins to know the temperatures on the veins and then average temperature is found. Similarly, five points are marked not on the veins to measure the skin temperatures and average temperature is determined. Differences between temperatures on the veins and not on the veins are calculated.

It is observed that the average temperature on the veins is always higher by a couple of degrees than the adjacent tissues in normal conditions. Better contrasts in the form of larger difference of temperatures between veins and their surroundings are found after cold stimulations. When the difference of average temperatures reaches its maximum value then veins can be seen clearly. This analysis also helps in finding the optimum imaging time for IR thermography after cold stimulation. Evaluation of temperatures after stimulations is an important step for determining how effective the stimulations are in thermal imaging.

Table 2 presents all three thermal images of right dorsal hand of a female subject captured at the same time i.e. $25 \mathrm{~s}, 35 \mathrm{~s}$ and $45 \mathrm{~s}$ after $15 \mathrm{~s}$ long application of cold compress for three different scenarios of this study. Three images are taken with an interval of $10 \mathrm{~s}$ for each case. For each subject, a total of 9 infrared images of each hand are obtained and a total of 18 thermal images for both hands. After selecting the region of interest in all 9 images of every subject of each hand, a prominent vein/s is chosen.

Five (black coloured) markers are placed on the vein/s, as indicated in the thermal images presented in the Table 2 to calculate temperature on a particular vein/s. Eventually, the mean vein temperature is measured using five values. Another five (cyan coloured) markers are placed on the surrounding tissue and the mean skin tissue temperature is calculated. For more 
precision mean of the mean temperature of vein and the skin tissue are calculated through three images of each case. Consequently, the difference between the vein and neighbouring tissue temperature is determined and plotted. Better contrasts in the temperatures are obtained for pumping condition as compared to non-pumping. Without tourniquet scenario is regarded as a base or reference condition because there is no hindrance in the venous flow for this case. But thermal images of this condition help to pin point the veins and the neighbouring tissue because of good contrast and clear visibility. Otherwise it might be difficult to decide without these reference images to identify veins for pumping and non-pumping conditions especially in the difficult visibility subjects. Fig. 6 presents the average temperature difference values between the vein and the surrounding tissue temperatures for all 20 subjects and overall mean of the average temperature difference value for the entire study for left and right dorsal hands.

Table 2 Thermal images of right dorsal hand of a female subject with Vacuderm but with and without pumping, and without tourniquet. Black five $(1-5)$ markers are on the veins whereas, cyan five $(6-10)$ are not on the veins but on the surrounding tissue.

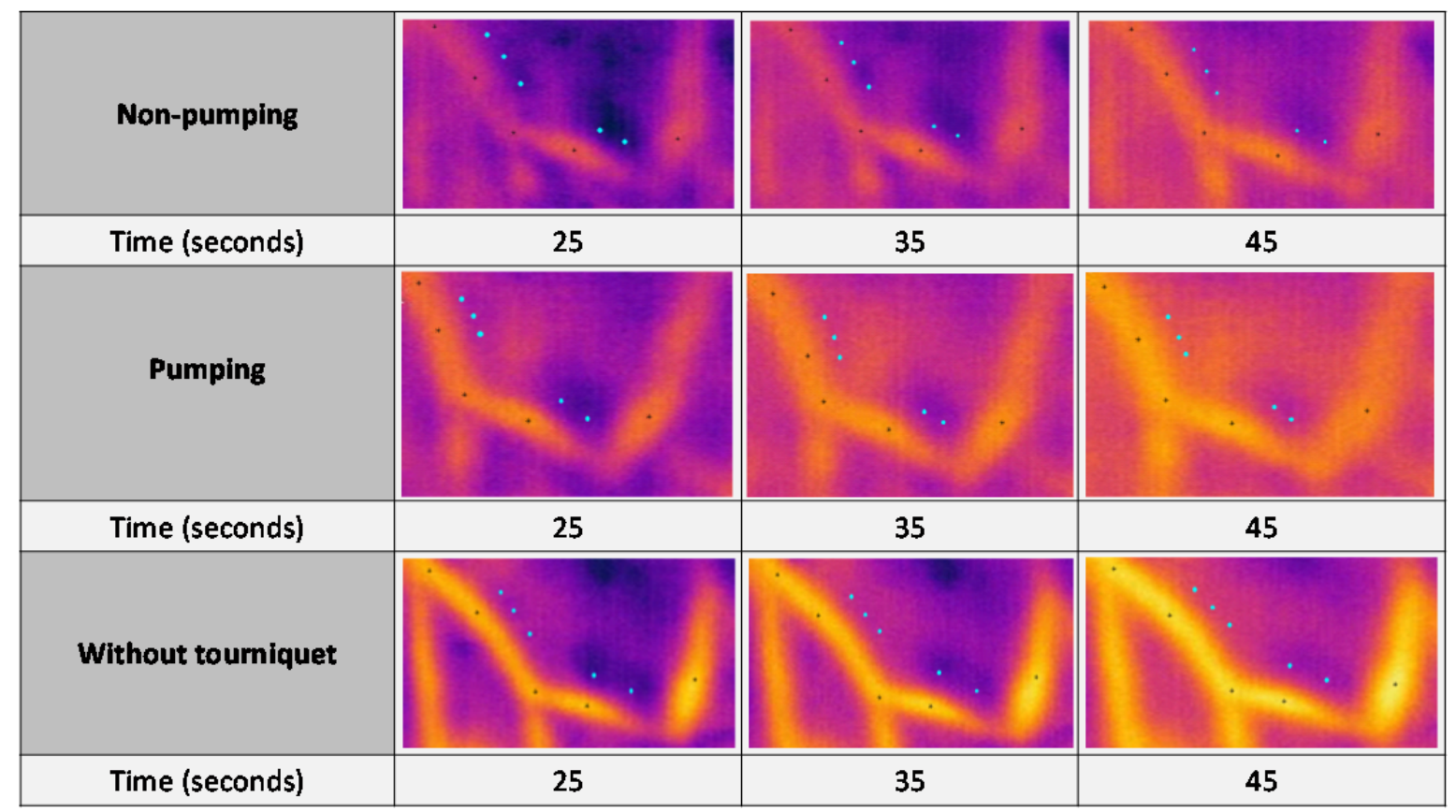

Fig. 4-c1 illustrates that in $70 \%$ participants the average temperature difference are found considerably higher after pumping effect of the Vacuderm in the case of the left dorsal hand. In the right dorsal hands, in $80 \%$ participants the average temperature difference are found 
remarkably higher after pumping effect of the Vacuderm (Fig. 4-c2). Fig. 4-c3 and Fig. 4-c4 clearly supports the argument on overall average temperature difference of entire sample size.

\section{Vein temperature analysis: paired samples t-tests}

Paired samples t-test about average temperature difference between vein and the surrounding tissue in case of left dorsal hands for non-pumping vs pumping conditions tells a very significant association between these two conditions. The mean score for left hand pumping condition is higher $(\mathrm{M}=2.09, \mathrm{SD}=.74)$ than for left hand non-pumping $(\mathrm{M}=1.62, \mathrm{SD}$ $=.77)$. There is a very significant statistical difference between these two conditions; $\mathrm{t}(19)=$ $-2.298, \mathrm{p}<0.05$.

Paired samples t-test about average temperature difference between vein and the surrounding tissue in case of right dorsal hands for non-pumping versus pumping conditions reveals a very significant association between these two conditions. The mean score for right hand pumping condition is higher $(\mathrm{M}=1.90, \mathrm{SD}=.71)$ than for right hand non-pumping $(\mathrm{M}=$ $1.36, \mathrm{SD}=.57)$. There is a very significant statistical difference between these two conditions; $\mathrm{t}(19)=-4.604, \mathrm{p}<0.001$. 


\section{Discussion}

Many organisations are currently active in addressing vascular access difficulties such as NIVAS $^{26}$ and the $\mathrm{NHS}^{27}$, via the development of an Integrated Care Pathways (ICP). This research is expected to help health care organisations and vascular access practitioners.

Results clearly indicate that pumping effect of the Vacuderm plays a significant role towards the vein visibility and its prominence. Due to pumping more veins become visually obvious as compared to non-pumping scenarios. Furthermore, temperature increases after pumping. We postulated that this is because pumping increases venous inflow into the more proximally occluded vein yielding it bigger in size and more pressurised. Higher temperature of the veins is also responsible for showing better contrast between vein and the adjacent tissue. The increased venous distension and tension is expected to translate into a more palpable vein and a better preparation of the vein for venous access as a result of pumping. As demonstrated statistically with pumping there is additional vein enlargement, and appear visually clearer on thermal images.

In summary, our hypothesis held true for vein visibility, prominence, significant increase in vein diameter and higher contrast between the veins and surrounding skin tissues as a result of the pumping effect of the Vacuderm tourniquet, but bigger diameter served as a better predictor of visibility than did temperature difference on visual analysis. Our data analyses have demonstrated the significant enhancement of vein visualising through the use of the Vacuderm.

The upper limbs are chosen in this study for capturing thermal images and analyses because these sites are easier to study logistically as subjects rolled their sleeves up. In literature, it is proved that the captured image of the back of the hand is of a good quality, providing more useful information as compared to the vein image at palm and wrist which are comparatively of poorer quality. ${ }^{25}$ The importance of the determination of vein width for selecting a vein appropriate for the cannulation procedure has been highlighted in literature. ${ }^{26}$ The best choice of the vein for catheterization is the one with maximum longitudinal area and widest in the diameter. As demonstrated here the Vacuderm helps to dilate the vein through pumping with resultant increase in blood influx into a proximally occluded vein. The patients' characteristics also influence the results, although our results are in this respected limited by sample size to significantly assess these factors. However, the cross over design of the study 
and the use of both upper limbs has allowed the study to have good statistical power providing highly significant statistical results on vein size, clarity and temperature.

\section{Conclusion}

This study has investigated the physiological effect of a novel tourniquet, the Vacuderm, on the vein dimensions, visual clarity and vein temperature of distal upper limbs' veins through creating a negative pressure via manual pumping action in addition to the tourniquet effect using infrared thermography (IRT). Our findings contribute original, novel and apposite data on intravenous access. The chi-square statistics for vein visibility for left dorsal hands is 10.1403 with $p$-value of 0.006282 . Also, for the right dorsal hand $\chi 2=9.817$ and $p$-value $=$ 0.0074. The results for both dorsal hands are highly significant. Additionally, the results showed statistically a significant increase of $24-31 \%$ in the vein lumen diameter and 54 $70 \%$ in venous cross sectional area of left and right hands respectively as well as high statistically significant increase in the vein clarity as assessed by infrared thermography. Moreover, paired samples t-test about the average temperature difference between veins and the surrounding tissue in case of the right dorsal hands for non-pumping vs pumping conditions revealed a very significant association between these two conditions and the same results are found for the left hands. The visibility of the veins, making them eligible for cannulation, consistently increased due to increase in the temperature difference between the vein and neighbouring tissue when using the pumping effect of the new medical device, the Vacuderm after the application of cold compress. This is anticipated to improve the ease of venous access when pumping of the Vacuderm is performed in addition to its tourniquet effect. As a result of this physiological change in veins, practitioners from all disciplines and every level of experience are expected to be supported to overcome the difficulties of peripheral IV catheter placement. Fewer attempts and obstacles translate to lower financial burdens for hospitals. But most importantly, a reduction in failed placements means a reduction in unnecessary trauma and danger for all patients who require IV therapy or cannulation. The routine application of a standard tourniquet is clearly safe procedure and it works in a similar way by expanding the vein. Therefore, vein expansion is safe as part of the procedure for cannulation. The Vacuderm is meant to enhance this expansion for patient with DiVA.

Overall, the findings agree with our hypothesis, showing the efficacy of the added pumping action (introducing vacuum or negative pressure) of an innovative tourniquet, the Vacuderm in increasing venous size and clarity to the eye and expected improved palpability. The health 
professionals, such as doctors and nurses may consider this new device an expedient resource for vein identification, detection and preparation when they deal with the difficult intravenous access cases.

\section{Funding and Declaration of Interest}

This study is partially funded by Olberon Ltd; but none of the authors work for Olberon Ltd or have any commercial interest in the product. 


\section{References}

1. Shaw SJ. Locating difficult veins for venepuncture and cannulation. Nursing Standard. 2017 Feb 15;31(25):62-71.

2. Alexandrou E, Ray-Barruel G, Carr PJ, Frost S, Inwood S, Higgins N, Lin F, Alberto L, Mermel L, Rickard CM. International prevalence of the use of peripheral intravenous catheters. Journal of hospital medicine. 2015 Aug 1;10(8):530-3.

3. Sebbane M, Claret PG, Lefebvre S, Mercier G, Rubenovitch J, Jreige R, Eledjam JJ, de La Coussaye JE. Predicting peripheral venous access difficulty in the emergency department using body mass index and a clinical evaluation of venous accessibility. The Journal of emergency medicine. $2013 \mathrm{Feb}$ 28;44(2):299-305.

4. Hallam C, Weston V, Denton A, Hill S, Bodenham A, Dunn H, Jackson T. Development of the UK Vessel Health and Preservation (VHP) framework: a multi-organisational collaborative. Journal of Infection Prevention. 2016 Mar;17(2):65-72.

5. Wallis MC, McGrail M, Webster J, Marsh N, Gowardman J, Playford EG, Rickard CM. Risk factors for peripheral intravenous catheter failure: a multivariate analysis of data from a randomized controlled trial. Infection Control \& Hospital Epidemiology. 2014 Jan;35(1):63-8.

6. McManus, C., Alexandrou, E., Ale, J., Mifflin, N., Frost, S.A. and Sou, V., 2017. A clinical pathway for the management of difficult venous access. BMC nursing, 16(1), p.64.

7. Carr, P.J., Rippey J.C., Budgeon C.A., Cooke M.L., Higgins N. and Rickard C.M., 2016. Insertion of peripheral intravenous cannulae in the emergency department: factors associated with first-time insertion success. Journal of Vascular Access, 17(2), pp.182-190.

8. Dougherty, L., 2013. Intravenous therapy in older patients. Nursing Standard, 28(6), pp.50-58.

9. Maiocco G, Coole C. Use of ultrasound guidance for peripheral intravenous placement in difficult-toaccess patients: advancing practice with evidence. Journal of nursing care quality. 2012 Jan 1;27(1):515.

10. Fields JM, Piela NE, Ku BS. Association between multiple IV attempts and perceived pain levels in the emergency department. The journal of vascular access. 2014;15(6):514-8.

11. Helm RE, Klausner JD, Klemperer JD, Flint LM, Huang E. Accepted but unacceptable: peripheral IV catheter failure. Journal of Infusion Nursing. 2015 May 1;38(3):189-203.

12. Oliver, G. ed., 2015. Whose line is it anyway? British Journal Nursing. 24, Suppl 2, S3. 
13. Royal College of Nursing, 2016. Standards for Infusion Therapy. 4th ed. [booklet] London: RCN.

14. Roberge RJ, Kelly M, Evans TC, Hobbs E, Sayre M, Cottington E. Facilitated intravenous access through local application of nitroglycerin ointment. Annals of emergency medicine. 1987 May 1;16(5):546-9.

15. Mbamalu D, Banerjee A. Methods of obtaining peripheral venous access in difficult situations. Postgraduate medical journal. 1999 Aug 1;75(886):459-62.

16. Costantino TG, Parikh AK, Satz WA, Fojtik JP. Ultrasonography-guided peripheral intravenous access versus traditional approaches in patients with difficult intravenous access. Annals of emergency medicine. 2005 Nov 30;46(5):456-61.

17. Dargin JM, Rebholz CM, Lowenstein RA, Mitchell PM, Feldman JA. Ultrasonography-guided peripheral intravenous catheter survival in ED patients with difficult access. The American journal of emergency medicine. 2010 Jan 31;28(1):1-7.

18. Roberge RJ. Venodilatation techniques to enhance venepuncture and intravenous cannulation. The Journal of emergency medicine. 2004 Jul 31;27(1):69-73.

19. Chiao FB, Resta-Flarer F, Lesser J, Ng J, Ganz A, Pino-Luey D, Bennett H, Perkins Jr C, Witek B. Vein visualization: patient characteristic factors and efficacy of a new infrared vein finder technology. British journal of anaesthesia. 2013 Feb 5;110(6):966-71.

20. Galloway S, Bodenham A. Ultrasound imaging of the axillary vein-anatomical basis for central venous access. British Journal of Anaesthesia. 2003 May 1;90(5):589-95.

21. Pirotte T, Veyckemans F. Ultrasound-guided subclavian vein cannulation in infants and children: a novel approach. British Journal of Anaesthesia. 2007 Mar 1;98(4):509-14.

22. Olberon 2017, The Vacuderm ${ }^{\mathrm{TM}}$. Available at: http://www.olberon.com/vacuderm. [Accessed 15 July 2017].

23. Asrar M, Al-Habaibeh A, Houda M. Innovative algorithm to evaluate the capabilities of visual, near infrared, and infrared technologies for the detection of veins for intravenous cannulation. Applied optics. 2016 Dec 1;55(34):D67-75.

24. Deng ZS, Liu J. Enhancement of thermal diagnostics on tumors underneath the skin by induced evaporation. InEngineering in Medicine and Biology Society, 2005. IEEE-EMBS 2005. 27th Annual International Conference of the 2006 Jan 17 (pp. 7525-7528). IEEE.

25. Xueyan L, Shuxu G. The fourth biometric-vein recognition. InPattern recognition techniques, technology and applications 2008. InTech. 
26. Shahzad A, Walter N, Malik AS, Saad NM, Meriaudeau F. Multispectral venous images analysis for optimum illumination selection. InImage Processing (ICIP), 2013 20th IEEE International Conference on 2013 Sep 15 (pp. 2383-2387). IEEE.

27. NIVAS National Infusion and Vascular Access Society, https://nivas.org.uk/, accessed on 5 October 2018.

28. NHS, National Health Service, https://www.nhs.uk/, accessed on 5 October 2018. 


\section{Appendix: Supplemental Materials}

Table A1 Demographic profile with subjects' characteristics and ambient temperatures before and after the experimental procedure

\begin{tabular}{|c|c|c|c|c|c|c|c|c|c|}
\hline ID & Gender* & $\begin{array}{l}\text { Skin } \\
\text { Tone } \text { e }^{* *}\end{array}$ & $\begin{array}{l}\text { Weight } \\
\text { (kg) }\end{array}$ & $\begin{array}{l}\text { Height } \\
\text { (cm) }\end{array}$ & $\begin{array}{c}\text { Body } \\
\text { Mass } \\
\text { Index } * * * \\
\end{array}$ & $\begin{array}{c}\text { Age } \\
\text { (year) }\end{array}$ & $\begin{array}{c}\text { Ambient } \\
\text { Temperature } \\
\text { Before T1 }\left({ }^{\circ} \mathrm{C}\right) \\
\end{array}$ & $\begin{array}{c}\text { Ambient } \\
\text { Temperature } \\
\text { After T2 }\left({ }^{\circ} \mathrm{C}\right) \\
\end{array}$ & $\begin{array}{c}\text { Temperature } \\
\text { Difference } \\
\Delta \mathrm{T}\left({ }^{\circ} \mathrm{C}\right) \\
\end{array}$ \\
\hline 1 & 1 & 2 & 71.0 & 174 & 2 & 37 & 24.7 & 24.7 & 0.0 \\
\hline 2 & 2 & 3 & 73.0 & 178 & 2 & 21 & 25.0 & 25.5 & 0.5 \\
\hline 3 & 1 & 1 & 98.0 & 169 & 4 & 29 & 25.0 & 24.9 & 0.1 \\
\hline 4 & 1 & 2 & 84.0 & 183 & 3 & 29 & 24.3 & 23.9 & 0.4 \\
\hline 5 & 1 & 2 & 120.0 & 181 & 4 & 48 & 23.8 & 24.0 & 0.2 \\
\hline 6 & 2 & 2 & 72.0 & 153 & 4 & 36 & 24.2 & 24.7 & 0.5 \\
\hline 7 & 1 & 2 & 88.0 & 170 & 4 & 50 & 24.4 & 24.5 & 0.1 \\
\hline 8 & 1 & 1 & 70.0 & 178 & 2 & 27 & 24.3 & 24.7 & 0.4 \\
\hline 9 & 1 & 1 & 103.0 & 185 & 4 & 23 & 24.6 & 24.5 & 0.1 \\
\hline 10 & 1 & 2 & 115.0 & 188 & 4 & 49 & 25.8 & 24.5 & 1.3 \\
\hline 11 & 2 & 3 & 72.0 & 173 & 2 & 27 & 25.7 & 25.3 & 0.4 \\
\hline 12 & 1 & 3 & 78.0 & 174 & 3 & 27 & 25.8 & 25.7 & 0.1 \\
\hline 13 & 2 & 1 & 47.0 & 169 & 1 & 27 & 25.9 & 25.2 & 0.7 \\
\hline 14 & 1 & 1 & 93.0 & 188 & 3 & 27 & 25.7 & 25.2 & 0.5 \\
\hline 15 & 2 & 3 & 54.0 & 167 & 2 & 23 & 25.3 & 25.7 & 0.4 \\
\hline 16 & 1 & 1 & 54.4 & 161 & 2 & 24 & 21.9 & 21.5 & 0.4 \\
\hline 17 & 1 & 3 & 91.0 & 175 & 3 & 24 & 21.7 & 21.4 & 0.3 \\
\hline 18 & 2 & 1 & 70.0 & 169 & 2 & 25 & 24.0 & 24.3 & 0.3 \\
\hline 19 & 2 & 1 & 64.5 & 169 & 2 & 23 & 25.1 & 23.7 & 1.4 \\
\hline 20 & 2 & 1 & 72.0 & 160 & 3 & 34 & 24.5 & 23.6 & 0.9 \\
\hline
\end{tabular}

"Gender: 1 = "Male", 2 = "Female"

* Skin Tone: 1 = "Fair", 2 = "Olive", 3 = "Dark"

*** Body Mass Index: 1 = "Underweight", 2 = "Healthy weight", 3 = "Overweight", 4 = "Obese" 
Table A2 Infrared images data presentation of right hand of a female and left hand of a male for all three scenarios.

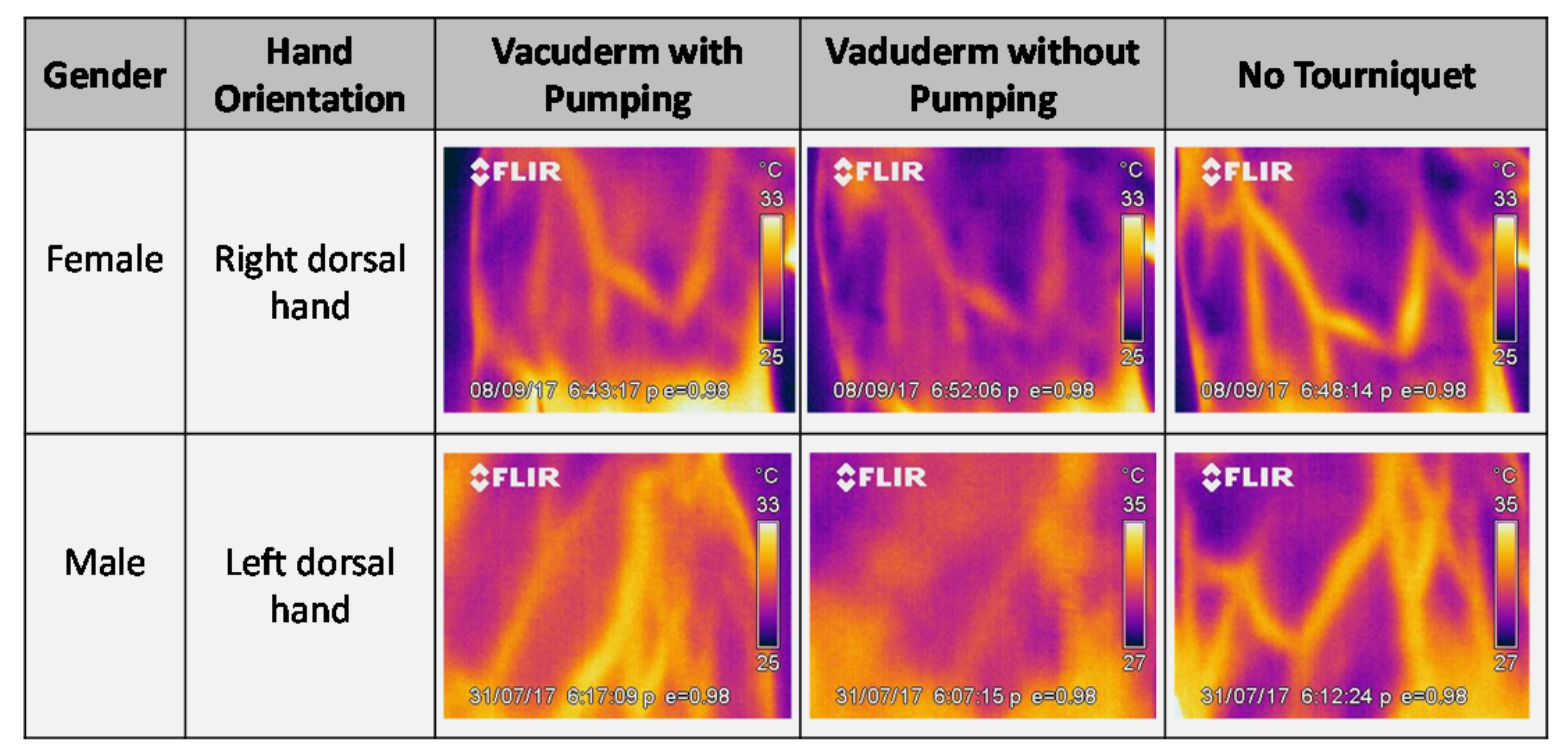


Table A3 Vein clarity scale or grades

\begin{tabular}{|c|c|c|c|c|c|c|}
\hline $\begin{array}{c}\text { Subject } \\
\text { ID }\end{array}$ & $\begin{array}{l}\text { Left Hand } \\
\text { Non- } \\
\text { pumping } \\
\end{array}$ & $\begin{array}{l}\text { Left Hand } \\
\text { Pumping }\end{array}$ & $\begin{array}{c}\text { Left Hand } \\
\text { Without } \\
\text { Tourniquet } \\
\end{array}$ & $\begin{array}{c}\text { Right Hand } \\
\text { Non- } \\
\text { pumping } \\
\end{array}$ & $\begin{array}{c}\text { Right Hand } \\
\text { Pumping }\end{array}$ & $\begin{array}{c}\text { Right Hand } \\
\text { Without } \\
\text { Tourniquet } \\
\end{array}$ \\
\hline 1 & 3 & 1 & 1 & 2 & 1 & 2 \\
\hline 2 & 2 & 3 & 1 & 2 & 1 & 1 \\
\hline 3 & 2 & 1 & 1 & 1 & 2 & 1 \\
\hline 4 & 2 & 3 & 1 & 2 & 2 & 1 \\
\hline 5 & 2 & 1 & 2 & 2 & 1 & 2 \\
\hline 6 & 2 & 1 & 2 & 2 & 1 & 1 \\
\hline 7 & 3 & 2 & 2 & 1 & 1 & 1 \\
\hline 8 & 3 & 1 & 1 & 2 & 2 & 1 \\
\hline 9 & 1 & 1 & 1 & 2 & 1 & 1 \\
\hline 10 & 2 & 1 & 1 & 3 & 2 & 1 \\
\hline 11 & 2 & 1 & 1 & 2 & 1 & 1 \\
\hline 12 & 3 & 2 & 1 & 2 & 1 & 1 \\
\hline 13 & 3 & 1 & 1 & 3 & 2 & 1 \\
\hline 14 & 2 & 1 & 1 & 3 & 2 & 1 \\
\hline 15 & 3 & 2 & 1 & 3 & 2 & 1 \\
\hline 16 & 3 & 2 & 3 & 3 & 2 & 2 \\
\hline 17 & 3 & 2 & 1 & 2 & 1 & 2 \\
\hline 18 & 1 & 2 & 1 & 2 & 2 & 1 \\
\hline 19 & 3 & 1 & 2 & 3 & 2 & 1 \\
\hline 20 & 1 & 1 & 1 & 1 & 1 & 1 \\
\hline
\end{tabular}

